Check for updates

Cite this: Nanoscale Adv., 2019, 1, 3537

\title{
Generation and enhancement of surface acoustic waves on a highly doped p-type GaAs substrate
}

\begin{abstract}
Boqun Dong (D) and Mona E. Zaghloul
Surface acoustic waves (SAWs) have been widely studied due to their unique advantage to couple the mechanical, electrical, and optical characteristics of semiconductor materials and have successfully been used in many industrial applications. In this work, we report a design that uses piezoelectric material Zinc Oxide $(\mathrm{ZnO})$ to enhance the generation and propagation of SAWs on the surface of a highly doped $\mathrm{p}$ type Gallium Arsenide (GaAs) substrate, which is more extensively used in optoelectronic devices than intrinsic GaAs structures. To maximize the piezoelectricity and successfully generate SAWs, high quality $\mathrm{c}$-axis orientation of the $\mathrm{ZnO}$ film is needed; thus we experiment and develop optimized recipes of a radio frequency (RF) magnetron sputtering system to deposit $\mathrm{ZnO}$ on the GaAs substrate. To further optimize the SAW performance, an intermediate Silicon Oxide $\left(\mathrm{SiO}_{2}\right)$ layer is added between the $\mathrm{ZnO}$ film and GaAs substrate. Additionally, we test samples with varied thickness of $\mathrm{ZnO}$ films and dimensions of interdigital transducer (IDT) fingers to figure out their individual effect on SAW properties. The results and techniques demonstrated in this paper will provide guidance for further studies on enhancing SAWs propagating along many other doped semiconductor materials. This combination of acoustics and optoelectronics in doped semiconductors is a promising start to building enhanced and hybrid devices in various fields.
\end{abstract}

Received 4th May 2019

Accepted 14th July 2019

DOI: 10.1039/c9na00281b

rsc.li/nanoscale-advances intrinsic GaAs devices to build hybrid structures, generating SAWs on doped GaAs has not been achieved so far.

Doping of GaAs is commonly used in applications in the optoelectronic field and is an essential method for achieving better photoelectric performance. Therefore, improving the compatibility between doped GaAs and SAWs has been an important research topic in order to widely extend the application range of SAWs in optical and electronic devices. To achieve the generation and propagation of SAWs on the doped GaAs surface, two key factors need to be considered and resolved. First, the piezoelectric coupling coefficient of GaAs is much smaller than those of commonly used piezoelectric materials such as $\mathrm{ZnO}, \mathrm{LiNbO}_{3}$ and AlN; thus only weak SAWs can be generated. ${ }^{\mathbf{1 3 - 1 5}}$ Second, when a piezoelectric substrate, in this case, GaAs, has been doped, the concentration of free carriers increases, leading to an electrical screening of the radio frequency field applied on the IDTs, and results in a restraint of the generation of SAWs. ${ }^{7,16,17}$ To resolve this electrical screening issue, researchers such as J. Rudolph et $a .^{16}$ and A. Violante et $a .^{7}{ }^{7}$ both came up with a similar method to place the doped layer away from the surface layers where SAWs were generated and propagated. A recent solution from Yuan and his colleagues is to add a metal layer on top of undoped GaAs/AlGaAs heterostructures on an n-type doped GaAs substrate (note that the undoped GaAs/AlGaAs layers covered the whole n-doped GaAs, but the metal layer did not). They used this metal layer to electrically screen the
Department of Electrical and Computer Engineering, The George Washington University, 800 22nd St NW, Washington, DC, USA.E-mail:dongbq@gwmail.gwu.edu 
piezoelectric field of SAWs so that the SAWs can travel and reach the GaAs in a mechanical way. ${ }^{17}$

In this present work, it is the first time to report that a SAW is able to propagate along the uncovered surface of a highly doped p-type GaAs substrate. The method we used is to build a $\mathrm{ZnO} /$ $\mathrm{SiO}_{2}$ island between metal IDTs and the p-type doped GaAs substrate. A thin layer of $\mathrm{ZnO}$ is deposited via using a $\mathrm{RF}$ magnetron sputtering system. The recipe of RF sputtering is optimized to make sure that the deposited ZnO layer has a highquality $c$-axis oriented crystalline structure, which is crucial to successfully generate the SAW. With the existence of $\mathrm{ZnO}$, the strong piezoelectric coupling effect of $\mathrm{ZnO}$ is used to more efficiently convert the electrical energy into mechanical energy and pass to GaAs, thus increasing the intensity of SAWs on GaAs. ${ }^{13,18}$ Next, we investigated the effect and advantage of a $\mathrm{SiO}_{2}$ layer with different thicknesses embedded between the $\mathrm{ZnO}$ layer and GaAs substrate and found that $\mathrm{SiO}_{2}$ layers over the $800 \mathrm{~nm}$ thickness level can further improve the performance of SAWs on p-type GaAs. This is because the $\mathrm{SiO}_{2}$ layer works as a buffer layer that helps mask the crystalline surface of GaAs and improve the adherence, thus forming a better crystal orientation of the $\mathrm{ZnO}$ layer. ${ }^{19-21}$ In addition to the enhancement of piezoelectricity, this designed $\mathrm{ZnO} / \mathrm{SiO}_{2}$ island between metal IDTs and the p-type GaAs substrate also helps separate the applied radio frequency field from the free carriers doped in the GaAs substrate. After applying radio frequency AC voltage, the $\mathrm{ZnO}$ layer utilizes its strong piezoelectricity to convert the applied electrical energy to mechanical energy in the form of periodical lattice vibration, generating SAWs. ${ }^{18,22}$ Next, the SAWs penetrate the $\mathrm{ZnO} / \mathrm{SiO}_{2}$ island and reach the surface of the GaAs substrate, mechanically passing the energy and the periodical vibration to the crystal lattice of GaAs. ${ }^{23-25}$ As a result, the screening of the applied radio frequency electrical field caused by the free carriers in doped GaAs can be effectively reduced because of the separation. Furthermore, we also studied the dependence of SAW enhancement on varied thickness of the ZnO layer and different widths of IDT fingers, and were able to select appropriate dimensions to optimize the performance of SAWs propagating along the p-type GaAs surface.

\section{Experimental}

\subsection{Device description}

As shown in Fig. 1(a), the structure of our device from top to bottom consists of metal IDTs, a $\mathrm{ZnO}$ layer, a $\mathrm{SiO}_{2}$ buffer layer, and a highly doped p-type GaAs substrate. The metal IDTs in the SAW delay line are oriented along the [011] direction on p-type (100) GaAs. According to the literature, ${ }^{26,27}$ SAWs propagating along the [011] direction on GaAs are piezoelectric, while those along directions like [001] or [010] are not piezoelectric. As discussed in the Introduction, many optoelectronic devices of

(a)
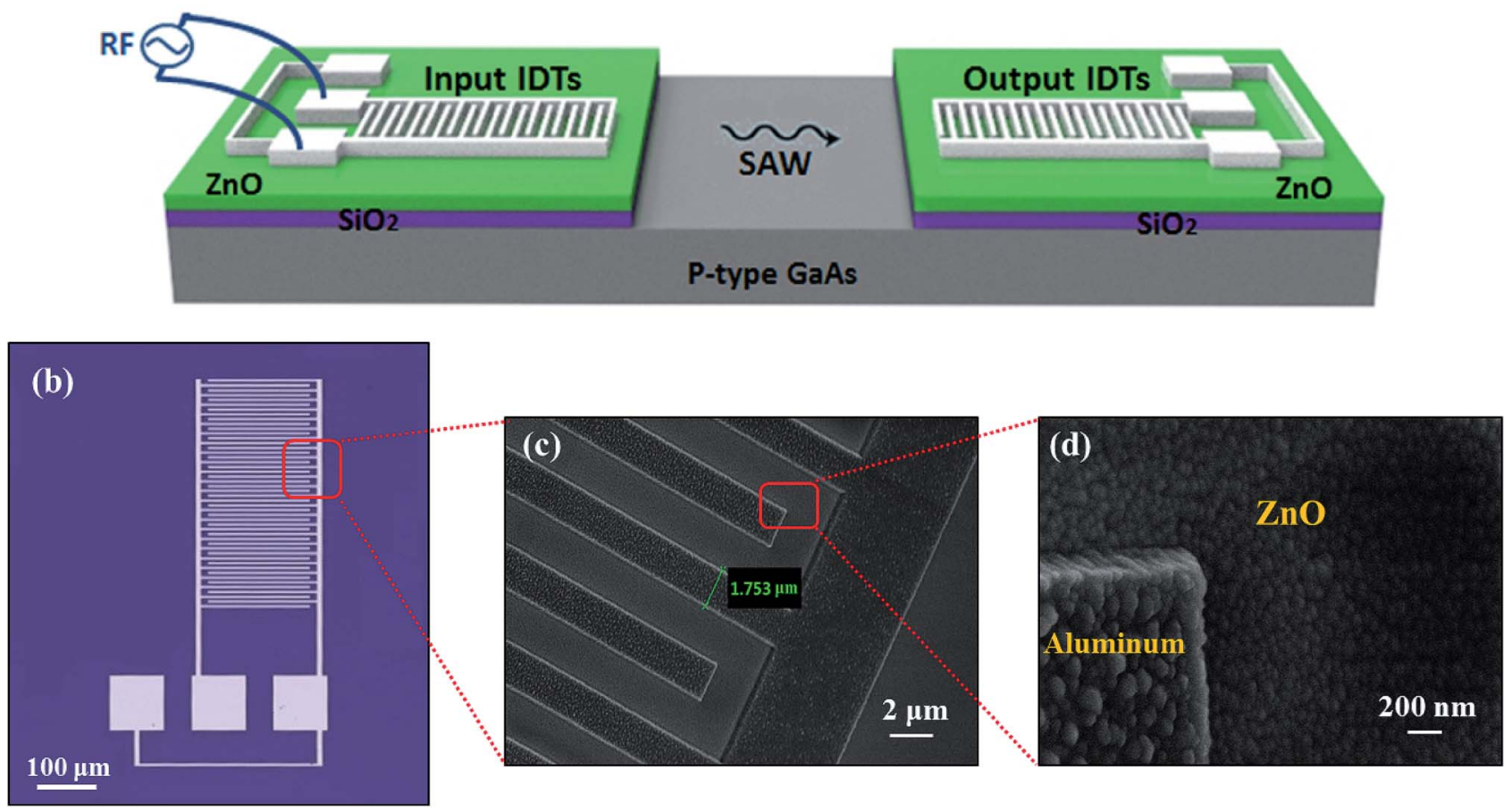

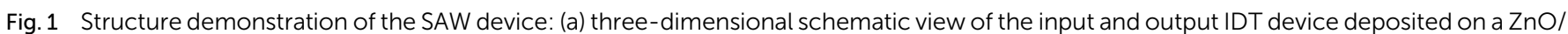

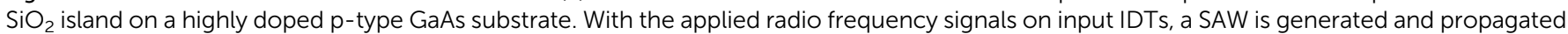

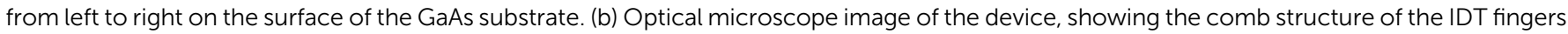

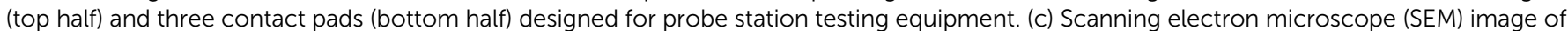

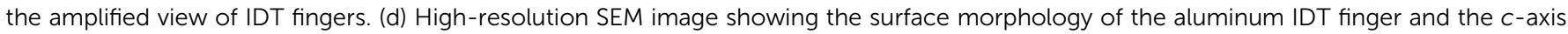
oriented $\mathrm{ZnO}$ layer. 
GaAs are expected to be modulated and improved by the piezoelectric fields induced by propagating SAWs. ${ }^{5-9}$ Based on this, we aim to enhance the propagating SAWs which are piezoelectric on GaAs in the present report to provide a foundation for further study. Therefore, we choose the [011] propagation direction on GaAs. The SAW is generated through the input metal IDTs which are present on the $\mathrm{ZnO} / \mathrm{SiO}_{2}$ island with the applied radio frequency signals. The SAW then propagates along the delay line (varied from $500 \mu \mathrm{m}$ to $2 \mathrm{~mm}$ ) on the surface of the p-type GaAs substrate. The output metal IDTs are used to receive the incoming SAW and to calculate the power transmission ratio.

The metal IDTs in our design are in the conventional singlefinger form. The spacing between IDT fingers is the same as the finger width. In this case, according to the concept and theory of SAWs ${ }^{18,28}$ the wavelength of SAWs is equal to four times the width of an IDT finger when the SAW is launched with the fundamental frequency of the IDT. As the propagating speed of the SAW in each specific material is known, the center frequency of the SAW can be determined as well. According to the literature, the SAW velocity in $\mathrm{ZnO}$ varies from $2600 \mathrm{~m} \mathrm{~s}^{-1}$ to $5000 \mathrm{~m} \mathrm{~s}^{-1}$ depending on the thickness and structure, ${ }^{15,29,30}$ and the SAW velocity in GaAs is approximately $2700 \mathrm{~m} \mathrm{~s}^{-1}$ to $2900 \mathrm{~m} \mathrm{~s}^{-1} \cdot{ }^{31,32}$ In our design, since the SAW completely propagates on the surface of the p-type GaAs substrate along the delay line (without the $\mathrm{ZnO}$ film), the center frequency is determined by the IDT finger width and the SAW velocity in GaAs.

The photos taken with an optical microscope (Fig. 1(b)) and scanning electron microscope (Fig. 1(c) and (d)) present a clear fabrication outcome and accurate dimensions. The metal IDT fingers are well shaped. The surface of the $\mathrm{ZnO}$ film is clean and smooth as needed for generating SAWs successfully.

\subsection{Sample fabrication}

The 3-dimensional schematic diagram in Fig. 2(a) shows the detailed fabrication process of the ZnO-enhanced SAW structure. The process started with using a highly doped p-type (100) GaAs wafer as the substrate. The doping concentration of the substrate ranges from $5 \times 10^{18} \mathrm{~cm}^{-3}$ to $3 \times 10^{19} \mathrm{~cm}^{-3}$. For the first stage of sample preparation, a standard cleaning process was applied with acetone and isopropyl alcohol (IPA), as well as using a sonicator if necessary. Next, we used the PECVD method with an optimized recipe to deposit $\mathrm{SiO}_{2}$ layers on top of the GaAs substrate at a rate of $120 \mathrm{~nm}$ per minute. Then we used a RF magnetron sputtering system to deposit a thin layer of $\mathrm{ZnO}$ on top of the $\mathrm{SiO}_{2}$ buffer layer.

For transferring the pattern, first a PMMA (A4) photoresist was spin-coated on top of the ZnO layer with a speed of

(a)

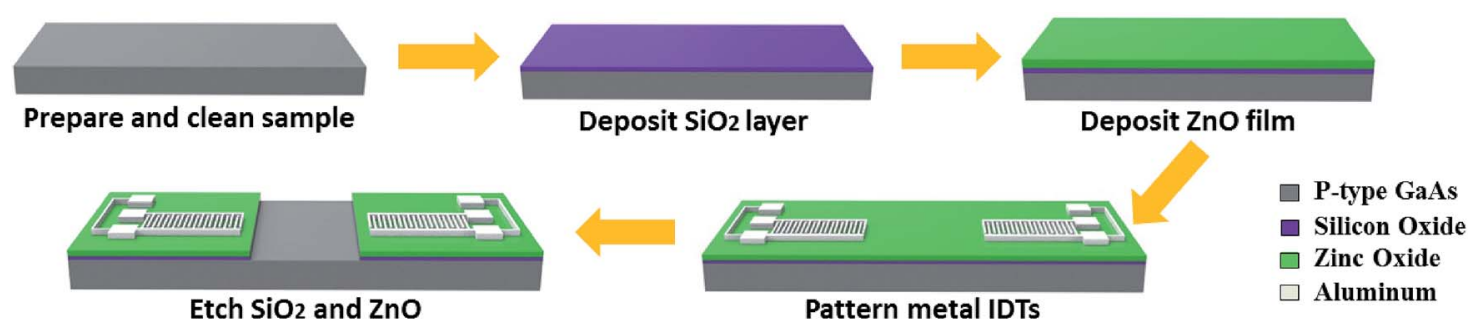

(b)

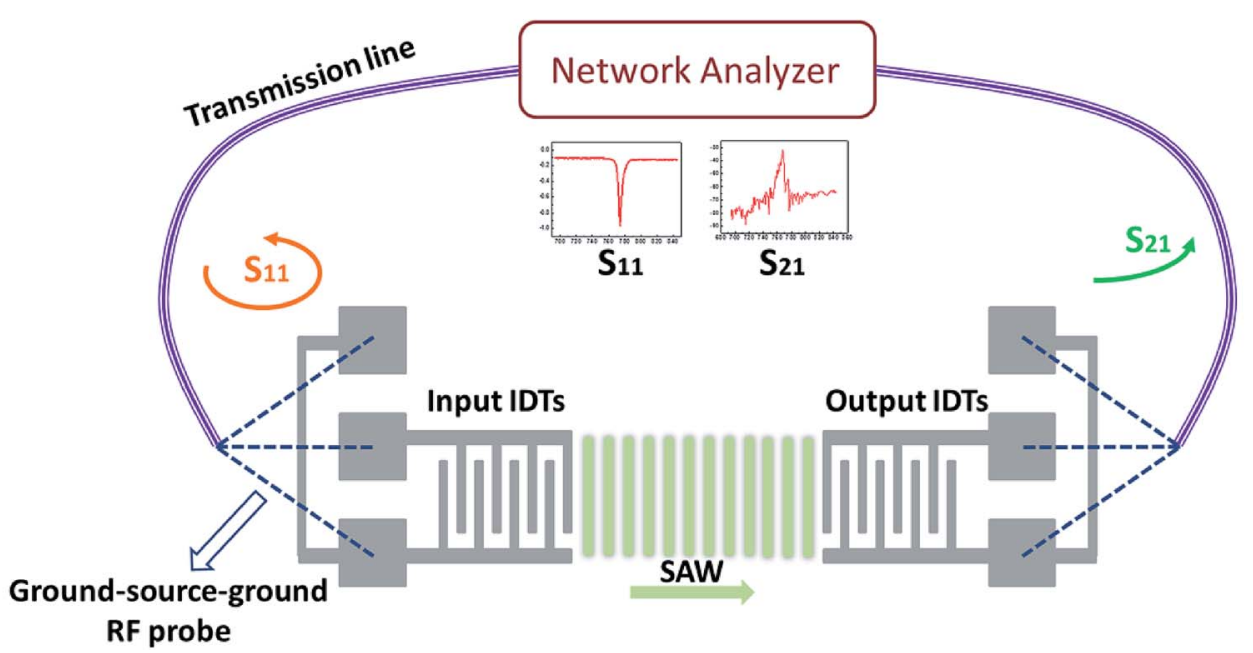

Fig. 2 (a) Fabrication process flow of the device: deposition of the $\mathrm{SiO}_{2}$ intermediate layer and $\mathrm{ZnO}$ thin layer, e-beam lithography and e-beam metal evaporation for aluminum IDTs, and etching of $\mathrm{SiO}_{2}$ and $\mathrm{ZnO}$ to open a window for the GaAs surface in the center. (b) Testing equipment: using RF probes and transmission line cables to connect the fabricated IDT device to a network analyzer to measure the transmission $\left(\mathrm{S}_{21}\right)$ and reflection $\left(\mathrm{S}_{11}\right)$ properties of SAWs. 
$3000 \mathrm{rpm}$ for two minutes. The sample was baked on a hot plate at a temperature of $180^{\circ} \mathrm{C}$ for 2 minutes. Then this spin-coating and heating process was repeated once more. Next, the process of ebeam exposure was conducted with a Raith Voyager Electron Beam Lithography (EBL) system to transfer the pattern onto the surface. The patterned photoresist was developed in methyl isobutyl ketone (MIBK) solution for 90 seconds. Then metallization with an e-beam evaporator was used to form the aluminum IDT electrode fingers. After that, the unwanted photoresist structure was lifted off with acetone for 20 minutes to get the IDT electrode fingers on top of $\mathrm{ZnO}$. The next step was to spin-coat another layer of the photoresist on top of the sample using the same method described previously. The process of e-beam exposure was conducted again with the Raith Voyager EBL system to transfer the second pattern on the top center of the sample, which was used to etch $\mathrm{ZnO} / \mathrm{SiO}_{2}$ and open a window as shown in Fig. 2(a). Afterwards, the photoresist was developed in MIBK solution for 90 seconds. Then the wet etching method and the reactive ion etcher (RIE) method were used to eliminate the patterned area of the $\mathrm{ZnO}$ layer and $\mathrm{SiO}_{2}$ layer, respectively, to create the window which has p-type GaAs exposed on the surface of the sample. The last step was to lift off the unwanted photoresist with acetone for 15 minutes to obtain the final structure of the device.

\subsection{Sample characterization and measurement}

The fabrication outcomes were characterized by using an optical microscope and scanning electron microscope (SEM) to make sure that the device structure and dimensions, thickness of layers, and surface morphology are achieved as designed. Xray diffraction measurements were conducted on the surface of the $\mathrm{ZnO}$ layer to verify the quality of RF sputtering deposition and to confirm the orientation of the deposited $\mathrm{ZnO}$ film.

Then, the sample with IDT devices was placed on a probe station. Both input IDTs and output IDTs are connected with an RF probe, separately. Each RF probe has three pins, which are ground-source-ground respectively. Thus we have three corresponding square contact pads on our IDT design, as shown in Fig. 2(b). Next, the two RF probes were connected to the two ports of a network analyzer via two transmission line cables. The network analyzer was used to apply scanning high frequency $\mathrm{RF}$ signals ranging from $50 \mathrm{MHz}$ to $2 \mathrm{GHz}$ between IDT fingers, and to measure the transmission and reflection characteristics (S-parameter) of SAWs. Here, we focused on the measurement of $S_{21}$ and $S_{11}$. The transmission coefficient $S_{21}$ refers to how much power is transmitted from input IDTs to output IDTs through the SAW. The reflection coefficient $S_{11}$ refers to how much power is reflected back from the input IDTs. As for the case of our single-finger IDT design (as shown in Fig. 1), when there is no SAW generated or weak SAW propagation, the $S_{21}$ indicates a big transmission loss and the $S_{11}$ is close to $0 \mathrm{~dB}$, suggesting that most of the power cannot be transmitted to the output. On the other hand, when a strong SAW is generated and propagated from the input to the output, the $S_{21}$ shows a peak at the center frequency with a bandwidth, and the $S_{11}$ has a dip in the same frequency range, as shown in the insets of Fig. 2(b).

\section{Results and discussion}

\subsection{Quality of the ZnO layer}

Before building devices following the fabrication process described above, it is essential to obtain a high quality deposition of ZnO layer. For a stronger piezoelectric effect, $\mathrm{ZnO}$ needs to be deposited with a high quality c-plane facing up when using the RF sputtering method, as SAWs cannot be generated and transmitted strongly on a low quality ZnO surface. The recipe of the RF magnetron sputtering system includes many parameters such as the plate temperature, gas flow ratio, VAT pressure, time length, pre-sputter and sputter power, and each has its impact on the deposition results.

Fig. 3(a) and (b) exhibit SEM pictures showing the top surface topography of the deposited $\mathrm{ZnO}$ layers. Both figures have the same magnification and thus good to compare. The two samples are ZnO layers deposited using different recipes in the RF magnetron sputtering system on two pieces of highly doped p-type GaAs substrates cut from the same wafer. Fig. 3(a) shows a low quality $\mathrm{ZnO}$ deposition with a very rough and cracked surface, making it difficult to pattern and deposit metal IDTs, and thus cannot be used to generate SAWs. After modifying parameters in the recipe, a high quality deposited $\mathrm{ZnO}$ layer is achieved with a smooth and clean surface and small ZnO grain size, as shown in Fig. 3(b).

The crystalline structure of the two deposited $\mathrm{ZnO}$ films using different RF sputtering recipes are also characterized using $\theta-2 \theta$ X-ray diffraction (XRD). Fig. 3(d) and (e) show XRD plots of low quality and high quality ZnO films, respectively. Upon comparing the two plots, it is clear that the high quality ZnO film has a much higher intensity of the (002) peak and a smaller number of off-oriented crystals. Also, the built-in lattice strain reduced from $1.369 \%$ to $0.512 \%$, and the full width at half maximum (FWHM) value of the (002) XRD reflection line reduced from 0.639 degree to 0.392 degree. These results prove that, with the modified recipe, a highly textured and highly $c$-axis oriented $\mathrm{ZnO}$ layer can be grown on the p-type GaAs substrate.

Fig. 3(c) and (f) show the outcome of the $\mathrm{ZnO}$ film deposited on a sample with an added $\mathrm{SiO}_{2}$ buffer layer using the same RF sputtering recipe for high-quality ZnO. According to the SEM image (Fig. 3(c)), the ZnO film exhibits a more visible hexagonal crystal structure with an increased grain size. Additionally, by comparing the XRD plots in Fig. 3(e) and (f), one can see that the higher intensity of the (002) peak is achieved with the help of the $\mathrm{SiO}_{2}$ buffer layer. Also, the built-in lattice strain reduced from $0.512 \%$ to $0.437 \%$, and the FWHM value of the (002) XRD reflection line reduced from 0.392 degree to 0.325 degree. These results confirm that a better crystal orientation of high-quality $\mathrm{ZnO}$ along the $c$-axis direction can be formed with a $\mathrm{SiO}_{2}$ buffer layer added between the $\mathrm{ZnO}$ film and p-type GaAs substrate.

\subsection{Enhancement of SAWs by ZnO}

Next, metal IDTs were deposited on top of the high quality $c$-axis oriented $\mathrm{ZnO}$ layer. To verify that the $\mathrm{ZnO}$ film is able to 
(a)

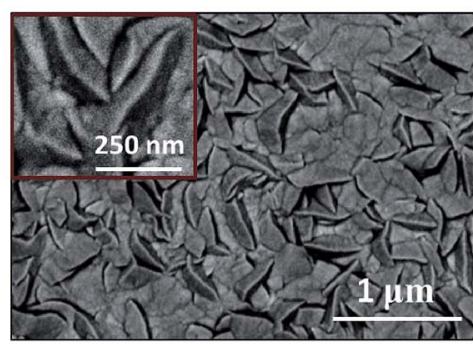

(d)

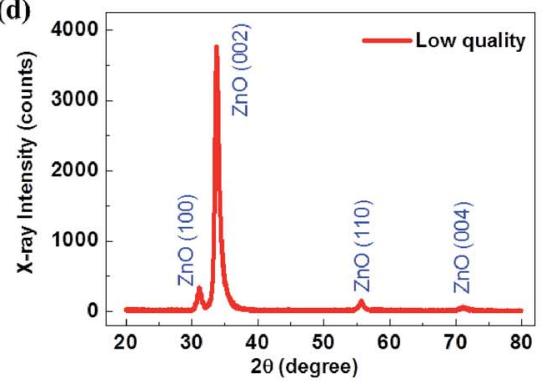

(b)

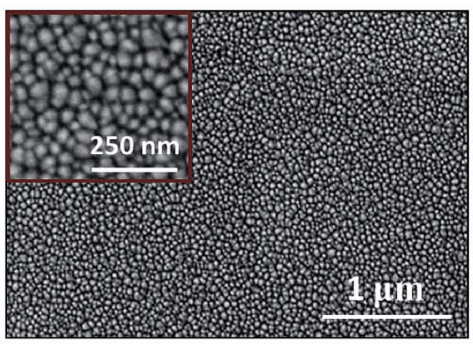

(e)

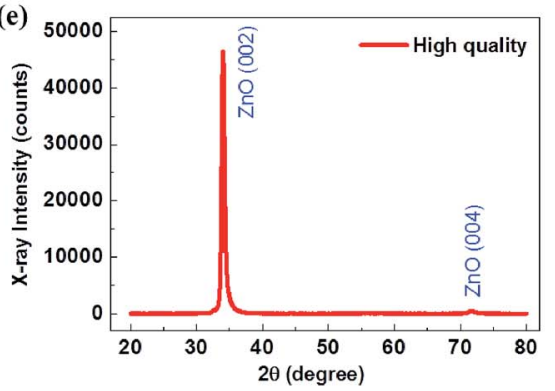

(c)

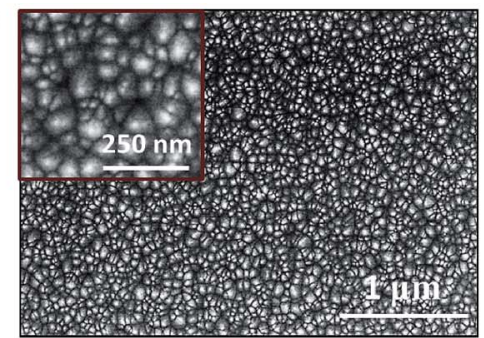

(f)

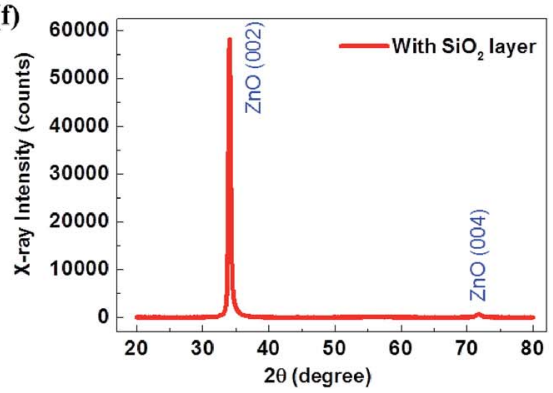

Fig. 3 Quality of ZnO layer deposition using the RF magnetron sputtering method: (a) top surface SEM image and (d) X-ray diffraction pattern of the $\mathrm{ZnO}$ film (thickness: $1400 \mathrm{~nm}$ ) grown on the p-type GaAs substrate resulting in a low quality outcome. The sputtering conditions of this sample are: $P=350 \mathrm{~W}, T=130{ }^{\circ} \mathrm{C}, \mathrm{Ar} / \mathrm{O}_{2}=69 \mathrm{sccm} / 0 \mathrm{sccm}$, pressure $=17 \mathrm{mTorr}$, and $t=2$ hour. (b) Top surface SEM image and (e) X-ray diffraction pattern of a high-quality c-axis oriented $\mathrm{ZnO}$ film (thickness: $1100 \mathrm{~nm}$ ) grown on the p-type GaAs substrate. The sputtering conditions of this sample are: $P=400 \mathrm{~W}, T=250^{\circ} \mathrm{C}, \mathrm{Ar} / \mathrm{O}_{2}=50 \mathrm{sccm} / 11 \mathrm{sccm}$, pressure $=8 \mathrm{mTorr}$, and $t=2$ hour. (c) Top surface SEM image and (f) X-ray diffraction pattern of a high-quality c-axis oriented ZnO film (thickness: $1100 \mathrm{~nm}$ ) with the same sputtering conditions as (b and e), but grown on a sample with an additional $\mathrm{SiO}_{2}$ buffer layer. The insets on the top left in (a-c) show the amplified view of each corresponding SEM image.

enhance the SAW propagation on the p-type GaAs surface, we tested different samples. One was an IDT structure deposited on the p-type GaAs substrate with an added ZnO layer, and the other one was without the $\mathrm{ZnO}$ layer. The comparison result of the transmission characteristics (S-parameter $\mathrm{S}_{21}$ ) of SAWs measured by using a network analyzer is shown in Fig. 4(d).

Fig. 4(a) shows the cross sectional view of the ZnO layer deposited on the p-type GaAs substrate. The lower black area is the GaAs substrate. The upper grey area shows the top surface of the $\mathrm{ZnO}$ layer, which is the same as the high quality c-plane $\mathrm{ZnO}$ shown in Fig. 3(b). The middle area is the fractured surface of the ZnO layer. The view of the fractured surface shows a dense and columnar structure, which once again verifies the high quality of the $c$-axis oriented crystalline structure of the $\mathrm{ZnO}$ film. The green line and number are used to measure and indicate the thickness of the $\mathrm{ZnO}$ layer, which is $1.42 \mu \mathrm{m}$ in this sample.

In Fig. 4(d), the red line is the transmission coefficient $S_{21}$ of the SAW generated by IDTs put on the surface of p-type GaAs. The blue line shows the result of the sample that has a ZnO layer added on top of p-type GaAs. The black line shows the result when there is no SAW propagation. According to the concept introduced in Section 2.1, the center frequency of the SAW in this case is determined by four times the IDT finger width (3.6 $\mu \mathrm{m})$ and the SAW velocity in GaAs $\left(\sim 2800 \mathrm{~m} \mathrm{~s}^{-1}\right)$, which gives $f_{\mathrm{SAW}} \sim 770 \mathrm{MHz}$ by calculation. The measurement result shown in Fig. 4(d) is consistent with this theoretical expectation. By comparison, the peak value of $S_{21}$ at the center frequency of the SAW increased from $-100 \mathrm{~dB}$ to $-75 \mathrm{~dB}$ on p-type GaAs before and after generating SAWs. This result shows the very weak piezoelectric coupling effect of the highly p-doped GaAs substrate. Then with the enhancement brought about by the added $\mathrm{ZnO}$ layer, the peak value of $\mathrm{S}_{21}$ increased further to -55 $\mathrm{dB}$. According to this result, the insertion loss becomes smaller, indicating that a stronger SAW is generated and propagated on the p-type GaAs surface as a result of the added $\mathrm{ZnO}$ film. On the other hand, not only the peak value, the $S_{21}$ in the whole measurement range around the center frequency increased. This can be explained by the existence of higher-order harmonics and other pseudo-surface waves and shear waves, ${ }^{30,33}$ which together contribute to the frequency response in the whole measurement range, especially for a basic IDT design without reflectors. ${ }^{21}$ In other words, when the piezoelectric coupling effect is enhanced, not only the SAW at the center frequency gets stronger, but also a wide range of frequency response increases, as seen in the literature. ${ }^{17,21,30,33}$ This wide-range increase of frequency response is also observed in Fig. 4(e) and (f), which is discussed in Section 3.3 and Section 3.4 , respectively. The measurement result presented in this section proves that a strong piezoelectric material such as $\mathrm{ZnO}$ can be used to improve the performance of SAWs on highly doped p-type GaAs.

\subsection{Intermediate layer of $\mathrm{SiO}_{2}$}

The next step was to use a $\mathrm{SiO}_{2}$ buffer layer to further improve the design. According to the literature, an intermediate layer of $\mathrm{SiO}_{2}$ deposited between GaAs and $\mathrm{ZnO}$ will effectively mask the crystalline surface of GaAs and thus allow good adherence and better texture quality of the $\mathrm{ZnO}$ deposition..$^{20,21} \mathrm{~A}$ higher quality 

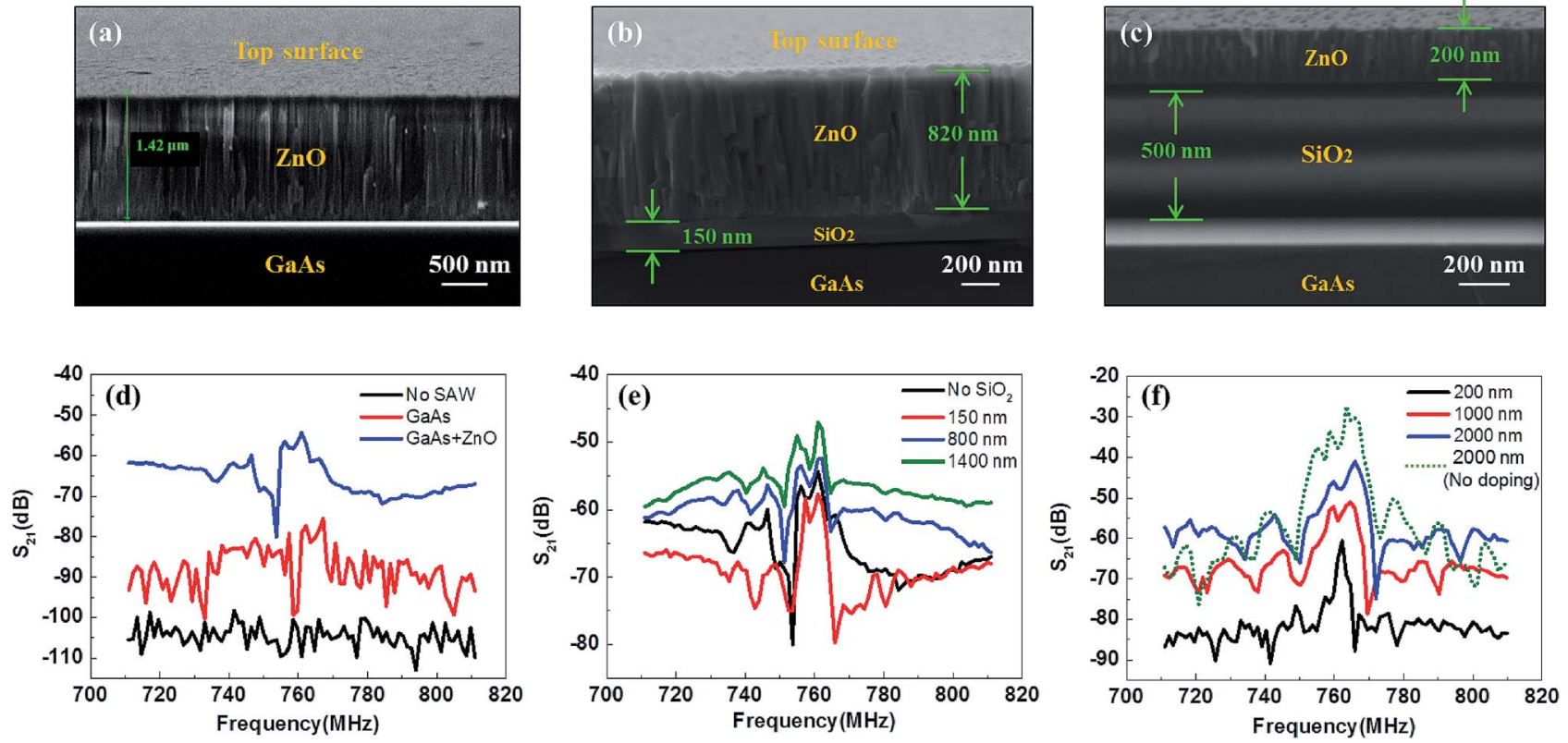

Fig. 4 Experimental results on samples with different layer structures: (a) SEM image of the cross-sectional view of the ZnO layer deposited on top of the p-type GaAs substrate without a buffer layer of $\mathrm{SiO}_{2}$. (b) $\mathrm{SEM}$ image of the cross-sectional view of a sample that has a $\mathrm{ZnO} / \mathrm{SiO} / \mathrm{GaAs}$ layer structure. (c) SEM image of the cross-sectional view of a sample that has a thicker $\mathrm{SiO}_{2}$ buffer layer and thinner $\mathrm{ZnO}$ film. (d) Comparison of the transmission property $\mathrm{S}_{21}$ of SAWs generated by IDTs deposited on the p-type GaAs substrate with and without an added ZnO layer. (e) Comparison of the transmission performance of SAWs generated on samples with an intermediate $\mathrm{SiO}_{2}$ layer of different thicknesses. (f) The three solid curves are comparison results showing the dependence of SAW enhancement on the thickness of ZnO films. The green dotted curve is the result from a sample fabricated on an undoped GaAs substrate. All experimental and test results presented in this section are conducted on single-finger IDTs with a finger width of $0.9 \mu \mathrm{m}$. The SAW delay line between each pair of input IDTs and output IDTs has the same distance (800 $\mu \mathrm{m})$ and [011] direction.

of the ZnO film will lead to enhanced piezoelectric coupling and improved SAW performance. To verify whether an intermediate layer of $\mathrm{SiO}_{2}$ is able to enhance the SAW, multiple samples with an added $\mathrm{SiO}_{2}$ layer were fabricated and tested. A comparison of the transmission performance of SAWs is presented in Fig. 4(e).

During the fabrication of this design, first we used the PECVD method to deposit $\mathrm{SiO}_{2}$ layers with varied thicknesses on different p-type GaAs substrates. Then $\mathrm{ZnO}$ films were deposited with the same thickness $(800 \mathrm{~nm})$ on top of $\mathrm{SiO}_{2}$ on those samples using the RF magnetron sputtering method. Fig. 4(b) shows the SEM picture showing the cross-sectional view of a thin $\mathrm{SiO}_{2}$ layer added as an intermediate layer between $\mathrm{ZnO}$ and GaAs. The material names in yellow color in Fig. 4(b) indicate different layers from bottom to top. The green lines with arrows and numbers are used to measure and indicate the thicknesses of the $\mathrm{ZnO}$ layer $(820 \mathrm{~nm})$ and $\mathrm{SiO}_{2}$ layer $(150 \mathrm{~nm})$. Fig. 4(c) shows the cross-sectional view of a thicker $\mathrm{SiO}_{2}$ layer $(500 \mathrm{~nm})$ embedded as an intermediate layer between a thinner ZnO film (200 nm) and p-type GaAs substrate.

From results shown in Fig. 4(e), compared to the sample without an intermediate $\mathrm{SiO}_{2}$ layer (black line), a thin layer of $\mathrm{SiO}_{2}(150 \mathrm{~nm}$, red line) results in a higher insertion loss, which is a sign of reduced performance of SAWs. The peak value of $\mathrm{S}_{21}$ at the center frequency of the SAW decreases from $-54 \mathrm{~dB}$ to -57 $\mathrm{dB}$, as well as the values in the rest of the frequency range. Then, with the increase of $\mathrm{SiO}_{2}$ thickness, the peak value at the center frequency of the SAW on the sample with an $800 \mathrm{~nm} \mathrm{SiO}$ layer (blue line) increases to $-52 \mathrm{~dB}$, which is a little bit higher than the one without $\mathrm{SiO}_{2}$ (black line). After continuously increasing the thickness of the $\mathrm{SiO}_{2}$ layer to $1400 \mathrm{~nm}$ (green line), the values of $\mathrm{S}_{21}$ in the whole frequency range appear clearly higher than the one without the $\mathrm{SiO}_{2}$ layer. Both the peak value at the center frequency $(-47 \mathrm{~dB})$ and the bandwidth are improved, which proves the enhancement of SAW transmission performance.

\subsection{Thickness of $\mathrm{ZnO}$}

On the other hand, in order to study the dependence of SAW enhancement on the thickness of the ZnO film, we fabricated and tested samples with different thicknesses of $\mathrm{ZnO}$ layers at $200 \mathrm{~nm}$, $1000 \mathrm{~nm}$ and $2000 \mathrm{~nm}$. We used the same recipe of RF magnetron sputtering and thus the orientation, quality and deposition rate of ZnO films were consistent for all samples. By setting different deposition time, the thickness of achieved $\mathrm{ZnO}$ films was varied. Besides the thickness of $\mathrm{ZnO}$ films, other parameters of the structure and dimensions (as shown in Fig. 4(c)) are the same for all samples as well. Measurement results of the transmission coefficient $\mathrm{S}_{21}$ of the different samples are shown in Fig. 4(f).

According to the comparison, both the peak value of $S_{21}$ (from $-60 \mathrm{~dB}$ to $-51 \mathrm{~dB}$ ) at the center frequency and the bandwidth of the SAW improve when increasing the thickness of the $\mathrm{ZnO}$ film from $200 \mathrm{~nm}$ (black line) to $1000 \mathrm{~nm}$ (red line). Then as the thickness of the ZnO film continues to increase to $2000 \mathrm{~nm}$ (blue line), the peak value of $S_{21}$ increases further to 
$-41 \mathrm{~dB}$ while the bandwidth does not increase much. This result shows a trend that a thicker $\mathrm{ZnO}$ film provides bigger enhancement of the SAW on the p-type GaAs substrate.

Additionally, in order to show the attenuation of the propagating SAW due to the free carriers doped in the GaAs substrate, we fabricated a SAW delay line on an undoped GaAs substrate with the same layer structure and IDT design, as well as all other parameters. The test result is plotted in Fig. 4(f) as a dotted green line. By comparing it to the solid blue line (both samples have a $2000 \mathrm{~nm} \mathrm{ZnO} \mathrm{film),} \mathrm{a} \mathrm{difference} \mathrm{of} 14 \mathrm{~dB}$ in the peak value of $\mathrm{S}_{21}$ (from $-41 \mathrm{~dB}$ to $-27 \mathrm{~dB}$ ) at the center frequency is found, while the bandwidths are close to each other. This SAW attenuation caused by the interaction of the piezoelectric field with the free carriers in the doped GaAs substrate is consistent with the expectation.

\subsection{Dimensions of IDTs}

As discussed in Section 2.1, the center frequency of the SAW is determined by the IDT dimensions and the SAW velocity in a specific material. When the material (GaAs) is known, the center frequency changes with the IDT dimensions. In order to observe this property in our design and also to find out the dependence of SAW performance on frequency, the study and comparison of IDTs with different sizes are given in this section. We fabricated two pairs of SAW devices with different IDT finger widths $(0.9 \mu \mathrm{m}$ and $2.3 \mu \mathrm{m})$ on the same $\mathrm{ZnO} / \mathrm{SiO}_{2} / \mathrm{GaAs}$ sample. Photos taken with an optical microscope shown in Fig. 5(a) and (e) are used to verify the outcomes of fabrication and the accuracy of IDT dimensions. The device structures of both IDT finger widths are well fabricated and their sizes are as desired.

Fig. 5(b) and (f) show photos taken with a SEM to give a magnified view of IDTs deposited on the $\mathrm{ZnO}$ surface. It is clear that the metal IDT fingers are well shaped and deposited during fabrication. The width of IDTs and the distance between two IDTs are $0.9 \mu \mathrm{m}$ and $2.3 \mu \mathrm{m}$ respectively as designed. The metal surface is a little rough due to the quality of chamber vacuum during the e-beam evaporation process. The $\mathrm{ZnO}$ surface, however, is smooth and clean as needed.

Curves plotted in Fig. 5(c) and (d) show the transmission properties (S-parameter $\mathrm{S}_{21}$ ) and reflection properties (Sparameter $S_{11}$ ) of the SAW generated through the $0.9 \mu \mathrm{m}$-width IDT device, respectively. The $x$-axis refers to the radio frequency range, and the $y$-axis refers to transmission loss and reflection loss in the unit of dB. Similarly, Fig. $5(\mathrm{~g})$ and $(\mathrm{h})$ show the plots of $\mathrm{S}_{21}$ and $S_{11}$ of the SAW generated by IDTs with a finger width of 2.3 $\mu \mathrm{m}$. From these plots, we can see that the SAWs are successfully generated and transmitted along the highly doped p-type GaAs surface in both cases. For the sample with the $0.9 \mu \mathrm{m}$ IDT finger, the center frequency of the SAW is $767 \mathrm{MHz}$ with an insertion loss $\left(S_{21}\right)$ of $-44.9 \mathrm{~dB}$ and the value of $\mathrm{S}_{11}$ is $-0.96 \mathrm{~dB}$. For the sample with the $2.3 \mu \mathrm{m}$ IDT finger, the center frequency of the SAW changed to $326 \mathrm{MHz}$. The transmission peak value of $\mathrm{S}_{21}$ decreased to $-57.1 \mathrm{~dB}$ and the reflection coefficient $\mathrm{S}_{11} \mathrm{dip}$ changed to $-0.95 \mathrm{~dB}$. This center frequency shift with the change of IDT sizes (from $0.9 \mu \mathrm{m}$ to $2.3 \mu \mathrm{m}$ ) is consistent with the theory that a smaller width of IDT fingers leads to a proportionally higher center frequency of SAW resonance in a specific piezoelectric material. It shows the capability of our design in propagating SAWs with different frequencies on highly doped p-type GaAs. In addition, by comparison between the two cases, we can see that the SAW with a higher frequency has better transmission performance on the p-type GaAs surface with the help of the $\mathrm{ZnO} / \mathrm{SiO}_{2}$ island design.

\section{Modelling and simulation}

To further verify that the $\mathrm{ZnO}$ thin film in our designed structure is capable of enhancing the piezoelectric coupling effect on
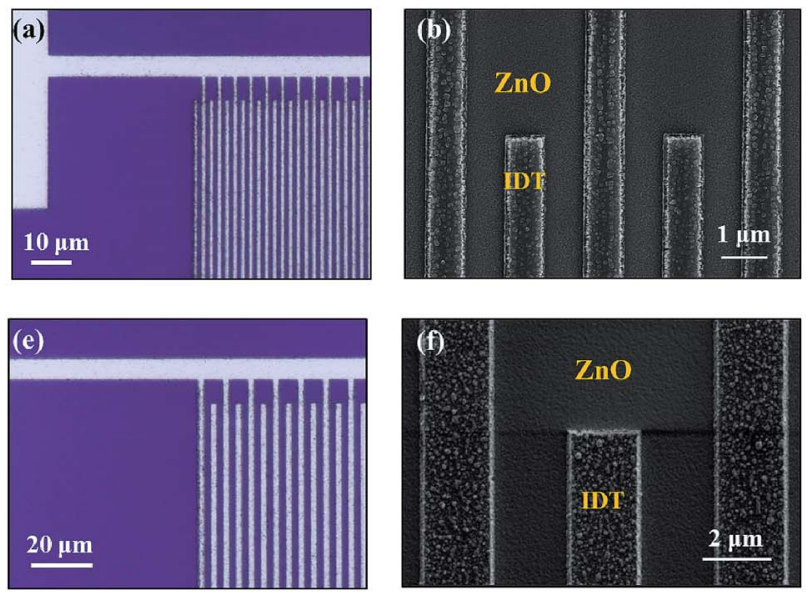
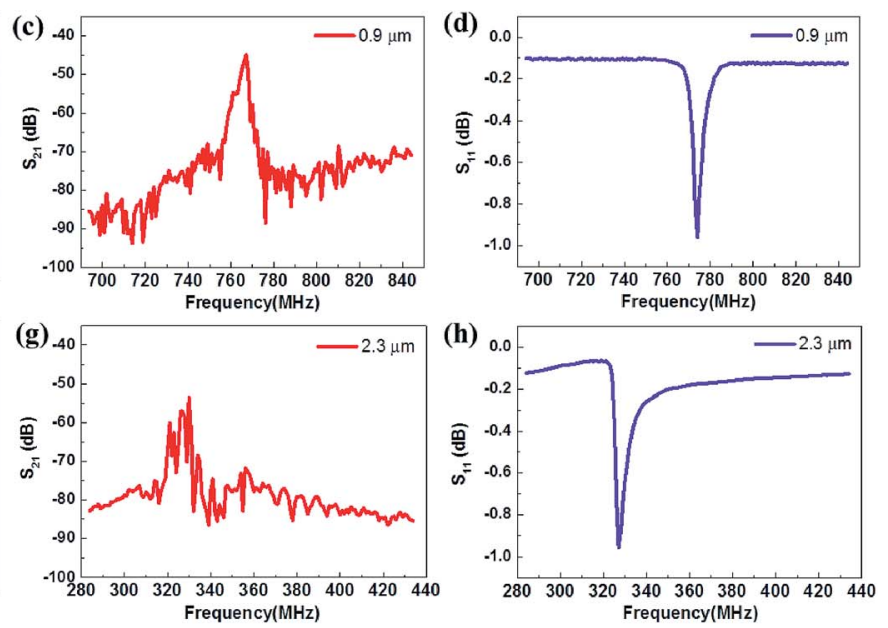

Fig. 5 Experimental results of devices with different IDT dimensions: (a) optical microscope image and (b) SEM image of the top surface view of IDT fingers placed on the $\mathrm{ZnO}$ film with a finger width of $0.9 \mu \mathrm{m}$. (c) Transmission characteristic $\mathrm{S}_{21}$ and (d) reflection characteristic $\mathrm{S}_{11}$ of $\mathrm{SAWs}$ generated by applied radio frequency AC voltage on $0.9 \mu \mathrm{m}$ IDTs. (e-h) Corresponding results for the device with IDT fingers of $2.3 \mu \mathrm{m}$ width. Both pairs of IDTs were fabricated on the same sample. They were designed to have the same acoustic impedance that can be matched as well as possible to the impedance of the network analyzer and the transmission line, which is close to $50 \Omega$. The delay line between each pair of input IDTs and output IDTs has the same distance $(1200 \mu \mathrm{m})$ and [011] direction. 
highly doped p-type GaAs and generating stronger SAWs, we used commercial simulation software COMSOL Multiphysics to do finite element analysis.

Fig. 6(b) shows the SAW generated on the ZnO substrate that has a strong piezoelectric coupling coefficient. On top of the ZnO layer, three pairs of IDTs made of aluminum are placed and used to generate periodically distributed piezoelectric fields. Among them, IDT no. 2, no. 4 and no. 6 are grounded, and IDT no. 1 , no. 3 and no. 5 are applied to AC input voltage that is expressed in the following equation:

$$
V_{\text {in }}=V_{0} \times \sin \left(2 \pi \times f_{0} \times t\right)
$$

where $V_{0}$ is chosen as 10 volts, and $f_{0}$ is set to $300 \mathrm{MHz}$ during simulation. The SAW is treated as a Rayleigh wave in this simulation, and the wave propagating speed is set to $2760 \mathrm{~m} \mathrm{~s}^{-1}$. Thus the wavelength of the SAW is calculated to be $9.2 \mu \mathrm{m}$. For the dimensions, the thickness of the substrate is $18.5 \mu \mathrm{m}$ and the width is $170 \mu \mathrm{m}$. The six IDTs are has a size of $2.3 \mu \mathrm{m} \times 2.3 \mu \mathrm{m}$. The distance between two IDTs is also $2.3 \mu \mathrm{m}$.

The results in Fig. 6(b) indicate the amount of surface deformation induced by SAWs. The separated areas in either blue color or red color suggest that the surface is shifted up and down, the same as the 3D schematic model shown in Fig. 6(a).
Next, GaAs is chosen as the substrate material. The (100) GaAs substrate is p-type doped with a doping concentration of 5 $\times 10^{18} \mathrm{~cm}^{-3}$. The IDTs are oriented along the [011] propagation direction. The piezoelectric coupling coefficient of GaAs is much smaller compared to those of commonly used piezoelectric materials. ${ }^{\mathbf{1 3 , 1 4}}$ As a result, the generated SAW is weak, as shown in Fig. 6(c).

In Fig. 6(c), the structure and dimensions of the substrate and IDTs as well as the applied AC voltage are completely the same as those designed in Fig. 6(b). The only difference is that the substrate material was changed from $\mathrm{ZnO}$ to GaAs. By comparing the two simulation results at the same instant of time ( $t=34.6 \mathrm{~ns})$, we can see smaller surface displacement in GaAs than that in ZnO. The maximum amount of displacement (in the vertical direction) on the $\mathrm{ZnO}$ substrate is about $0.2 \mathrm{~nm}$. For the GaAs substrate, however, the maximum amount of displacement is only around $0.004 \mathrm{~nm}$. This verifies that GaAs has a much weaker piezoelectric coupling effect than ZnO. Additionally, according to the SAW velocity used in simulation (also discussed in Section 2.1), the SAW should propagate from the IDT to the end (distance: $85 \mu \mathrm{m}$ ) within $31 \mathrm{~ns}$. As shown in Fig. 6(b), the SAW reaches the right and left ends of the structure when $t=34.6 \mathrm{~ns}$ as expected. However, for the GaAs

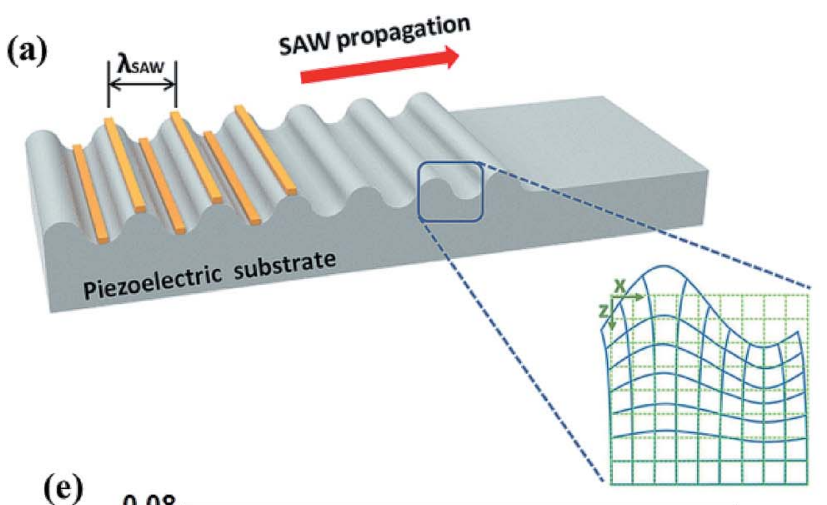

(e)

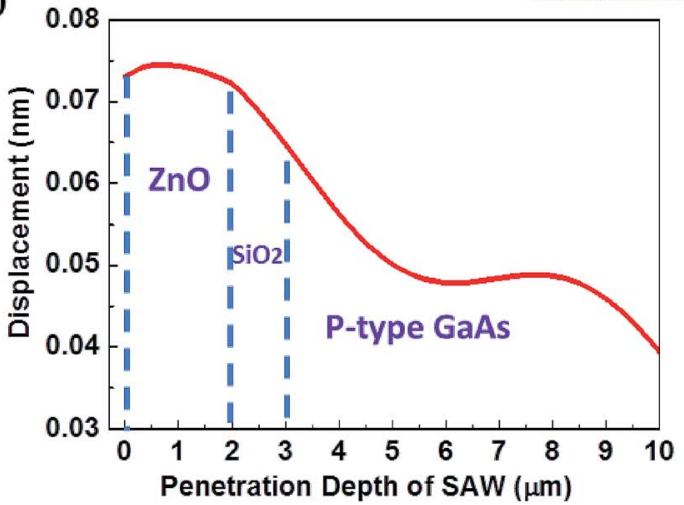

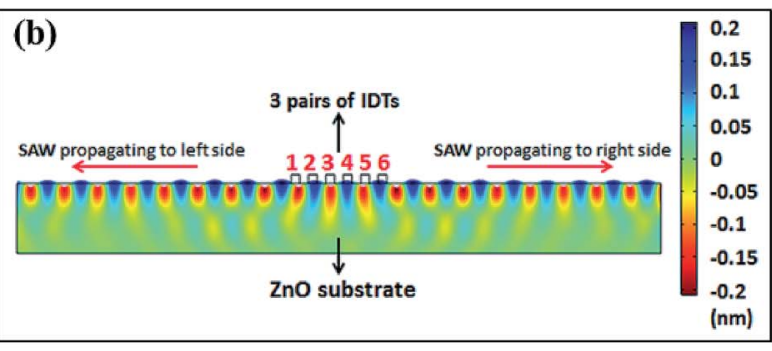
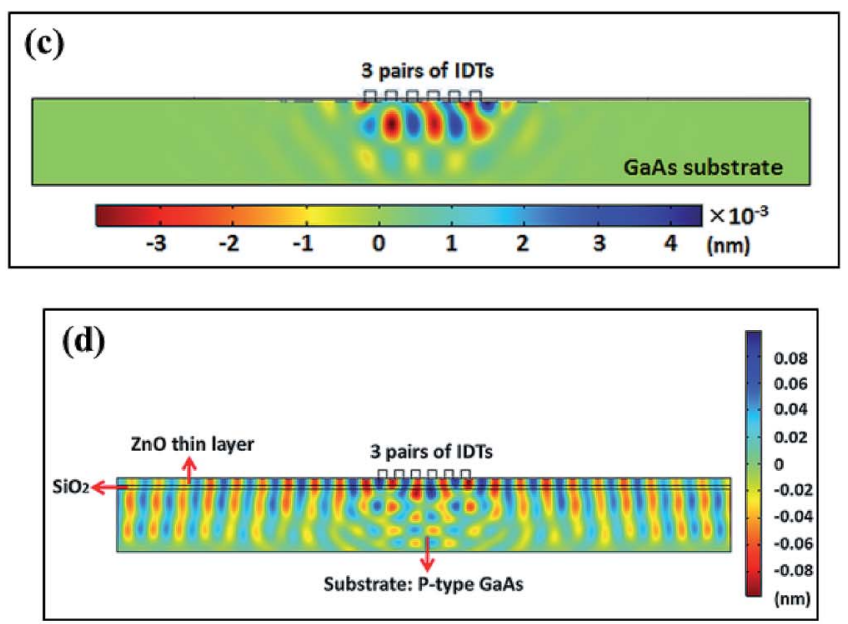

Fig. 6 Simulation of SAW enhancement for the $\mathrm{ZnO} / \mathrm{SiO}_{2} / \mathrm{GaAs}$ structure: (a) concept and mechanism of SAW propagation along the delay line and leading to surface deformations. The inset at the right corner shows the concept in a microscopic view that the crystal lattice is shifted up and down under the applied periodical piezoelectric potential and electric field. This, on the macroscopic scale, results in a periodical surface deformation and a propagating acoustic wave along the surface. The extent of surface displacement is in direct proportion to the built-in piezoelectric potential and electric field. Simulation results of surface deformations induced by SAWs that are generated and propagated on the (b) $\mathrm{ZnO}$ substrate, (c) GaAs substrate, and (d) $\mathrm{ZnO}$ thin film and $\mathrm{SiO}_{2}$ buffer layer placed on top of the GaAs substrate. (e) Simulation result to show the loss of SAW energy as the SAW penetrates from $\mathrm{ZnO} / \mathrm{SiO}_{2}$ into the $\mathrm{GaAs}$ substrate. 
substrate in Fig. 6(c), the SAW cannot visibly propagate that far at the same instant of time.

As discussed above, to enhance the piezoelectric coupling for p-type GaAs, a thin film of $\mathrm{ZnO}(2 \mu \mathrm{m})$ and a buffer layer of $\mathrm{SiO}_{2}$ $(1 \mu \mathrm{m})$ are placed on top of the GaAs substrate. The IDTs (width: $2.3 \mu \mathrm{m})$ are then placed on top of the $\mathrm{ZnO}$ layer. The [011] propagation direction of the SAW and other corresponding parameters are the same as those used in Fig. 6(b) and (c). Fig. 6(d) shows the simulation result of the new structure, which is captured at the same moment $t=34.6 \mathrm{~ns}$. According to this new simulation result, the generated SAW becomes much stronger when compared with the one shown in Fig. 6(c). The maximum amount of displacement in GaAs increases fifteen times from $0.004 \mathrm{~nm}$ to $0.06 \mathrm{~nm}$. In addition, the SAW is now able to propagate from IDTs to the ends of the right side and left side of the substrate surface. The curve in Fig. 6(e) is extracted and plotted from the simulation result of Fig. 6(d), showing the displacement amplitude induced by the SAW field along the penetration depth of the SAW in the vertical direction. It is used to indicate the energy loss when the SAW penetrates from $\mathrm{ZnO} /$ $\mathrm{SiO}_{2}$ into GaAs. As shown in Fig. 6(e), location $x=0$ refers to the top surface of the $\mathrm{ZnO}$ film, and then followed by the $\mathrm{SiO}_{2}$ layer $(2<x<3)$ and GaAs substrate $(x>3)$. It can be clearly seen that, even though an attenuation of the SAW field exists in the $\mathrm{ZnO} /$ $\mathrm{SiO}_{2}$ region, the displacement amplitude in GaAs (0.05-0.06 $\mathrm{nm}$ ) within a depth of several microns is much higher than the amount shown in Fig. 6(c). It verifies the SAW enhancement in p-type GaAs although some of the SAW energy was lost in the $\mathrm{ZnO} / \mathrm{SiO}_{2}$ region. These simulation results are consistent with the expected results described in theory and are identical to the experimental results we demonstrated in this paper, proving the function of our designed structure.

\section{Conclusion}

In this work, we studied and demonstrated a method of using a strong piezoelectric material $\mathrm{ZnO}$ with the $\mathrm{RF}$ magnetron sputtering deposition technique to enhance the piezoelectric coupling effect on the surface of a highly doped p-type GaAs substrate. Our presented technique has been proven successful in forming high quality $c$-axis oriented $\mathrm{ZnO}$ films and generating strongly enhanced SAWs that can propagate on p-type GaAs. Both the experimental results of the fabricated devices and the simulations verified this enhancement. Furthermore, we built and tested samples with intermediate $\mathrm{SiO}_{2}$ layers and found that an added $\mathrm{SiO}_{2}$ layer with an appropriate thickness can further improve the performance of SAWs. We also investigated the dependence of SAW properties on various factors such as the thickness of $\mathrm{ZnO}$ films and the sizes of IDT fingers. This work paves the way for further investigations of acoustoelectric properties of highly doped semiconductor materials and enables wider utilization of those materials in acoustic, electrical and optical applications.

\section{Conflicts of interest}

There are no conflicts to declare.

\section{Acknowledgements}

The research was partially supported by the U.S. Department of Energy (DOE) HEP STTR grant under award number DESC0017831. We gratefully appreciate the support from Dr Rolland Johnson and Muplus, Inc., and the nanofab facilities provided at the National Institute of Standards and Technology (NIST) and George Washington University (GWU). We also thank Dr Andrei Afanasev for the enlightening discussion and advice during the work.

\section{References}

1 S. Zhang, S. Lin, X. Li, X. Liu, H. Wu, W. Xu, P. Wang, Z. Wu, H. Zhong and Z. Xu, Nanoscale, 2016, 8, 226-232.

2 X. Li, S. Lin, X. Lin, Z. Xu, P. Wang, S. Zhang, H. Zhong, W. Xu, Z. Wu and W. Fang, Opt. Express, 2016, 24, 134-145.

3 B. Dong, S. Guo, A. Afanasev and M. Zaghloul, 2016, IEEE 43rd Photovoltaic Specialists Conference, PVSC, 2016, pp. 2087-2090.

4 S. Zhang, M. Poelker, M. L. Stutzman, X. Peng and J. Zou, Negative Electron Affinity Gallium Arsenide Photocathodes Based on Optically Resonant Nanostructure, Technical report, Thomas Jefferson National Accelerator Facility, Newport news, VA (United States), 2018.

5 C. Rocke, S. Zimmermann, A. Wixforth, J. Kotthaus, G. Böhm and G. Weimann, Phys. Rev. Lett., 1997, 78, 4099.

6 M. M. de Lima Jr and P. V. Santos, Rep. Prog. Phys., 2005, 68, 1639.

7 A. Violante, K. Cohen, S. Lazić, R. Hey, R. Rapaport and P. V. Santos, New J. Phys., 2014, 16, 033035.

8 B. Dong, S. Guo and M. Zaghloul, URSI Asia-Pacific Radio Science Conference, URSI AP-RASC, 2016, pp. 1921-1924.

9 S. Büyükköse, A. Hernandez-Minguez, B. Vratzov, C. Somaschini, L. Geelhaar, H. Riechert, W. G. van der Wiel and P. Santos, Nanotechnology, 2014, 25, 135204.

10 B. Dong, M. Zaghluol, A. Afanasev and R. P. Johnson, Proc. IPAC2018, Vancouver, BC, Canada, 2018.

11 A. Hernández-Mínguez, M. Möller, C. Pfüller, S. Breuer, O. Brandt, M. de Lima, A. García-Cristóbal, A. Cantarero, L. Geelhaar, H. Riechert, et al., MRS Online Proceedings Library Archive, 2012, p. 1408.

12 S. Hermelin, S. Takada, M. Yamamoto, S. Tarucha, A. D. Wieck, L. Saminadayar, C. Bäuerle and T. Meunier, J. Appl. Phys., 2013, 113, 136508.

13 Y. Kim, W. D. Hunt, F. S. Hickernell and R. J. Higgins, J. Appl. Phys., 1994, 75, 7299-7303.

14 E. D. Nysten, Y. H. Huo, H. Yu, G. F. Song, A. Rastelli and H. J. Krenner, J. Phys. D: Appl. Phys., 2017, 50, 43LT01.

15 A. Hernández-Mínguez, Y. Liou and P. Santos, J. Phys. D: Appl. Phys., 2018, 51, 383001.

16 J. Rudolph, R. Hey and P. Santos, Phys. Rev. Lett., 2007, 99, 047602.

17 M. Yuan, C. Hubert, S. Rauwerdink, A. Tahraoui, B. van Someren, K. Biermann and P. Santos, J. Phys. D: Appl. Phys., 2017, 50, 484004. 
18 C. C. Ruppel and T. A. Fjeldly, Advances in surface acoustic wave technology, systems and applications, World scientific, 2001, vol. 2 .

19 Y. Kim, W. D. Hunt, F. S. Hickernell, R. J. Higgins and C.-K. Jen, IEEE Trans. Ultrason. Eng., 1995, 42, 351-361.

20 K.-H. Bang, D.-K. Hwang and J.-M. Myoung, Appl. Surf. Sci., 2003, 207, 359-364.

21 W.-C. Shih and M.-S. Wu, J. Cryst. Growth, 1994, 137, 319325.

22 X. Du, Y. Fu, S. Tan, J. Luo, A. Flewitt, S. Maeng, S. Kim, Y. Choi, D. Lee, N. Park, et al., J. Phys.: Conf. Ser., 2007, 012035.

23 M. Gillinger, K. Shaposhnikov, T. Knobloch, M. Schneider, M. Kaltenbacher and U. Schmid, Appl. Phys. Lett., 2016, 108, 231601.

24 G. McHale, M. K. Banerjee, M. Newton and V. Krylov, Phys. Rev. B: Condens. Matter Mater. Phys., 1999, 59, 8262.
25 X. Du, Y. Q. Fu, S. Tan, J. Luo, A. Flewitt, W. Milne, D.-S. Lee, N.-M. Park, J. Park, Y. Choi, et al., Appl. Phys. Lett., 2008, 93, 094105.

26 A. Wixforth, J. Scriba, M. Wassermeier, J. Kotthaus, G. Weimann and W. Schlapp, Phys. Rev. B: Condens. Matter Mater. Phys., 1989, 40, 7874.

27 M. Chang, C. Lee, P. Asbeck, R. Vahrenkamp and C. Kirkpatrick, Appl. Phys. Lett., 1984, 45, 279-281.

28 V. M. Yakovenko, Phys. B, 2012, 407, 1969-1972.

29 J. X. Ding, D. Chen, L. Du and G. M. Liu, Applied Mechanics and Materials, 2012, pp. 1894-1898.

30 E. B. Magnusson, B. H. Williams, R. Manenti, M.-S. Nam, A. Nersisyan, M. J. Peterer, A. Ardavan and P. J. Leek, Appl. Phys. Lett., 2015, 106, 063509.

31 C. K. Campbell, Proc. IEEE, 1989, 77, 1453-1484.

32 C. Campbell, Surface acoustic wave devices and their signal processing applications, Elsevier, 2012.

33 T. Sato and H. Abe, IEEE Trans. Ultrason. Eng., 1998, 45, 394408. 